\title{
Aeroelastic tailoring using lamination parameters
}

\section{Drag reduction of a Formula One rear wing}

\author{
Glenn A. A. Thuwis • Roeland De Breuker • \\ Mostafa M. Abdalla · Zafer Gürdal
}

Received: 6 February 2009 / Revised: 15 June 2009 / Accepted: 5 September 2009 / Published online: 15 October 2009

(C) The Author(s) 2009. This article is published with open access at Springerlink.com

\begin{abstract}
The aim of the present work is to passively reduce the induced drag of the rear wing of a Formula One car at high velocity through aeroelastic tailoring. The angle-ofattack of the rear wing is fixed and is determined by the required downforce needed to get around a turn. As a result, at higher velocity, the amount of downforce and related induced drag increases. The maximum speed on a straight part is thus reduced due to the increase in induced drag. A fibre reinforced composite torsion box with extension-shear coupled upper and lower skins is used leading to bendingtorsion coupling. Three-dimensional static aeroelastic analysis is performed loosely coupling the Finite Element code Nastran and the Computational Fluid Dynamics panel code VSAERO using ModelCenter. A wing representative of Formula One rear wings is optimised for minimum induced drag using a response surface methodology. Results indicate that a substantial induced drag reduction is achievable while maintaining the desired downforce during low speed turns.
\end{abstract}

Keywords Aeroelastic tailoring - Lamination parameters . Drag reduction - Automotive

Part of this paper has been presented at the 2008 Sixth International Conference on Engineering Computational Technology in Athens (Thuwis et al. 2008).

G. A. A. Thuwis $(\bowtie) \cdot$ R. De Breuker $\cdot$ M. M. Abdalla $\cdot$ Z. Gürdal Faculty of Aerospace Engineering, TU Delft,

Kluyverweg 1, 2629 HS Delft, The Netherlands

e-mail: G.A.A.Thuwis@tudelft.nl

\section{Introduction}

In present Formula One (F1) racing, the continuous need for speed drives engineers to push both driver and the car to the limit. F1 cars nowadays regularly reach velocities up to $300 \mathrm{~km} / \mathrm{h}$ during a race. However, the cars are not optimised for high speed on straight lines, but are mostly optimised to achieve high turning speeds (Katz 2006). In order to be able to turn as fast as possible, the car must have as much traction as necessary to pass the turn at the intended velocity. At low speeds, this traction is mostly a mechanical traction originating from the car's weight acting on the tyres. At high speeds, however, the grip is mostly generated by the aerodynamic downforce. This downforce is provided by the aerodynamic surfaces and aerodynamic shaping of the bodywork (e.g. diffuser, front and rear wing). These aerodynamic devices give the driver the opportunity to go faster in a turn. The disadvantage is that, when the car is not cornering, the larger grip given by the aerodynamic devices, together with the increase in aerodynamic drag, tend to slow the car down.

At the dawn of Formula One racing, this problem was solved by means of moveable aerodynamic devices (Wright 1974). The driver had the ability to alter the angle-ofattack of the rear wing, such that on a straight line, the downforce and thus also the aerodynamic drag could be reduced. However, in the present regulations of the Fédération Internationale de l'Automobile (FIA 2007) it is stated that moveable aerodynamic devices are prohibited.

The question arises whether it is possible to design a fixed flexible wing capable of providing the right amount of downforce needed to turn at a certain velocity, while reducing the downforce at higher velocity when compared to a rigid wing. One such solution is presented in Massegur et al. (2007), where a two-element rear wing assembly is 
used with a flexible upper element. Due to its flexibility, the gap between both elements disappears at high velocities, causing the rear wing to reduce the produced downforce and induced drag.

In the present work, a concept for the reduction of induced drag is proposed based on aeroelastic tailoring of the rear wing. The rear wing contributes to one third of the total downforce (Zhang et al. 2006) and associated induced drag. Furthermore, tailoring the rear wing has no effect on any part of the car behind the rear wing, as would be the case for the front wing. A definition of aeroelastic tailoring within the context of Aerospace Engineering is given by Shirk et al. (1986):

\section{Aeroelastic tailoring is the embodiment of directional stiffness into an aircraft structural design to control aeroelastic deformation, static or dynamic, in such a fashion as to affect the aerodynamic and structural performance of that aircraft in a beneficial way.}

So, the target is to make use of the interaction between the structure and aerodynamics (Bisplinghoff et al. 1955; Dowell 2004) to design a rear wing such that at a high velocity the angle-of-attack, and as such the induced drag, of the rear wing are reduced. The wing design, at the same time, should still have the required angle-of-attack to generate sufficient downforce during cornering at low velocity. According to Lynch and Rogers (1976), aeroelastic tailoring creates the possibility of drag reduction compared to a rigid wing. More specifically, induced drag reduction using aeroelastic tailoring has been applied to improve Unmanned Aerial Vehicle (UAV) performance by Weisshaar et al. (1998), Bramesfeld et al. (2008), and to Micro Air Vehicles (MAV) in Stanford and Ifju (2008). According to Shirk et al. (1986) and Weisshaar and Duke (2006), wash-out wings are traditionally identified with induced drag reduction.

Composite laminate tailoring of the rear wing is proposed to obtain the envisioned result. The focus of this tailoring is on the torsion box of the rear wing (Rehfield and Cheung 2003, 2004; Guo et al. 2005, 2006). The effect of washout can be achieved by using an extension-shear coupled upper and lower skin (Fukunaga and Sekine 1994; Diaconu et al. 2002). The top and bottom skin of the torsion box are constructed to generate a circumferentially asymmetric stiffness (CAS) lay-up (Patil 1997). The net result of this construction is a bending-torsion coupling of the torsion box, without having bending-torsion coupling at the individual skin laminate level. This eliminates the curing problems encountered when bending-torsion coupling is present at laminate level. It has been shown by Weisshaar (1981) that bending-torsion coupling using a CAS lay-up is also effective for low aspect ratio wings.
The goal of this paper is to find a wing design which effectuates such a bending-torsion coupling to maximise the reduction in angle-of-attack of the rear wing at higher velocity while maintaining traction at low speeds. For this optimisation, the material properties are modelled using lamination parameters (Tsai and Pagano 1968), similar to previous work by Fukunaga and Vanderplaats (1991), Fukunaga and Sekine (1992), Miki and Sugiyama (1993), Abrate (1994), where lamination parameters are applied to model and optimise composite plates and shells, or wing structures in Liu and Haftka (2004). In Abdalla et al. (2007) and Kameyama and Fukunaga (2007), the lamination parameters are applied for aeroelastic tailoring of composite wing structures, and in Gürdal and Olmedo (1993) and Setoodeh et al. (2006), they are used to model variable stiffness composite plates.

In the following first a closer look at the problem at hand is presented. The third section presents a $2 \mathrm{D}$ aeroelastic analysis of a typical section to show the envisioned effect of bending-torsion coupling. This effect is further analysed using a 3D model which is presented in the consecutive section. This 3D model is used in a fluid-structure routine which is further described in section five. Finally the results are presented in the sixth section, followed by the conclusions and recommendations.

\section{Problem statement}

A Formula One car is a compromise between going as fast as possible on a straight track and achieving the highest possible turning speed while cornering. This problem is mostly solved, depending on the lay-out of the track, by optimising the car performance either to go fast through a corner or to go fast on a straight part of the circuit. It would, however, be a great achievement if the best of both worlds could be combined. In the following we analyse the cornering manoeuvre and the straight acceleration.

\subsection{Cornering manoeuvre}

To give a general idea about the maximum turn velocity, the car is represented as a rigid body having a mass $m$. Consider the force required to turn at a certain velocity:

$F^{r e q}=m \cdot \frac{V^{2}}{R}$,

where $V$ is the velocity along the path of the turn and $R$ the radius of that turn. This required force is generated through contact with the ground, where the maximum generated force is defined as:

$F^{\max }=(m \cdot g+L) \mu$, 


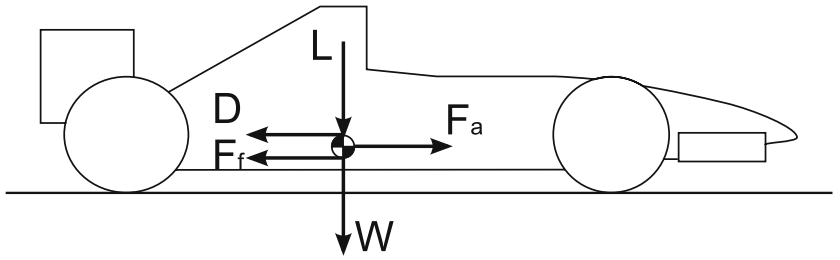

Fig. 1 Simplified representation of an F1 car

where $\mu$ is the friction coefficient of the tyres, $L$ is the downforce, and $g$ the gravitational acceleration. Using (1) and (2) and recalling $L=\frac{1}{2} \rho V^{2} C_{L} S$ results in the following expression for the maximum turning velocity:

$V=\left[\frac{1}{\mu g R}-\frac{\rho C_{L} S}{2 m g}\right]^{-\frac{1}{2}}$,

where $C_{L}$ is the 3D lift coefficient and $S$ the surface area of the rear wing. In order to increase the turning velocity, only $C_{L}$ can be changed in (3). The other constants are assumed to be fixed either by regulations $(m, S)$ or by external and physical factors $(\mu, R)$. From (3) it follows that the lift coefficient of the rear wing $C_{L}$ has to be increased in order to increase the turning velocity. Since in this work the focus is on the rear wing, only the rear wing's contribution to the lift coefficient is considered variable. Thus increasing the overall lift coefficient can be done by increasing the rear wing's angle-of-attack.

\subsection{Straight acceleration}

Since the angle-of-attack is a fixed value throughout a race, this high angle-of-attack also has an influence on the straight acceleration performance. Consider a basic representation of a car as depicted in Fig. 1, the total acceleration $a$ of the car is given by:

$a=\frac{F_{a}-F_{f}-D}{m}$,

where $F_{a}$ is an acceleration force given by the engine, $D$ is the aerodynamic drag force and $F_{f}$ is the friction force acting on the tyres. Since the engine is a fixed given subject, the acceleration force is assumed to be fixed as well. So to increase the acceleration of the car, both $F_{f}$ and $D$ are looked at. The friction force can be defined as: ${ }^{1}$

$F_{f}=\mu\left[m \cdot g+\frac{1}{2} \rho V^{2} S C_{L}\right]$.

\footnotetext{
${ }^{1}$ Note that this is a basic representation of the friction force using a constant friction coefficient. In reality this relation is much more complicated. However, for the purpose of demonstrating the influence on the acceleration a simple representation will suffice.
}

The drag force is defined as:

$D=\frac{1}{2} \rho V^{2} C_{D} S$

with $C_{D}$ the drag coefficient of the rear wing:

$C_{D}=C_{D_{0}}+\frac{C_{L}^{2}}{\pi R e}$,

where $C_{D_{0}}$ is the drag coefficient at zero angle-of-attack, $R$ is the aspect ratio of the rear wing, and $e$ the Oswald factor.

The increase in $C_{L}$ for the higher turning velocity will result in an increase of both the friction force $F_{f}$ in (5), and the drag force $D$ in (7). This leads to a lower overall straight acceleration of the car. The objective of this work is thus to obtain a flexible fixed wing which reduces both the friction force $F_{f}$, and the drag force $D$ at high velocity, by reducing the lift coefficient $C_{L}$ at this higher velocity. At the same time, however, the magnitude of the lift coefficient $C_{L}$ should not be reduced at low velocity in order to maximise the low speed turning velocity as defined in (3).

\section{Feasibility study}

In order to clarify the envisioned aeroelastic effect using bending-torsion coupling, a typical section is investigated using a static aeroelastic analysis. In Fig. 2 such a flat plate section is depicted, with $K_{h}$ and $K_{\alpha}$ the bending and torsional spring stiffness, respectively, $h$ the wing plunge, $c$ the chord length, and other parameters defined as:

$\alpha=\alpha_{0}+\alpha_{e}$

$C_{l}=C_{l_{\alpha}} \alpha$,

where $\alpha_{0}$ is the initial rigid angle-of-attack, $\alpha_{e}$ is the elastic angle-of-attack, $C_{l}$ is the $2 \mathrm{D}$ lift coefficient, and $C_{l_{\alpha}}=$ $2 \pi /(1+(2 / A R))$ for a finite flat plate. From this flat plate assumption it also follows that the moment around the aerodynamic center $m_{A C}$, and the lift coefficient at zero angle-of-attack $C_{l_{0}}$ are assumed to be zero.

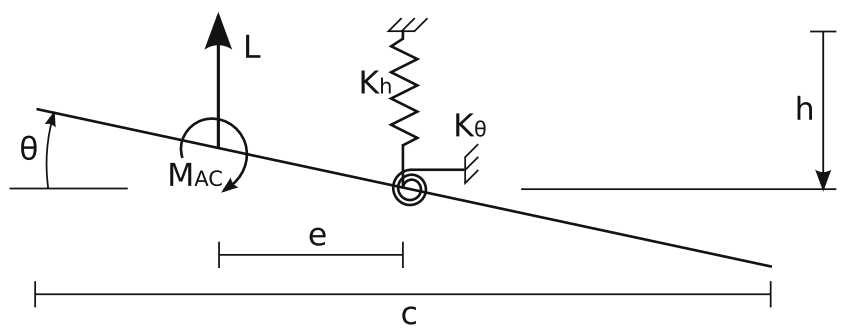

Fig. 2 2D section for aeroelastic analysis 
The moment about the elastic axis due to the aerodynamic loads is then defined as $m_{y}=L e$. Note that until now, the model does not have a coupling between the bending and torsion. This coupling is introduced using $K_{h \alpha}$ in the potential energy equation. Using Lagrange's equations (Török 2000), assuming static aeroelasticity and recalling $L=C_{l_{\alpha}}\left(\alpha_{0}+\alpha_{e}\right) c q$, the equations of motion become:

$\left\{\left[\begin{array}{cc}K_{h} & K_{h \alpha} \\ K_{h \alpha} & K_{\alpha}\end{array}\right]+c q\left[\begin{array}{cc}0 & C_{l_{\alpha}} \\ 0 & -C_{l_{\alpha}} e\end{array}\right]\right\}\left[\begin{array}{c}h \\ \alpha_{e}\end{array}\right]=c q \alpha_{0}\left[\begin{array}{c}-C_{l_{\alpha}} \\ C_{l_{\alpha}} e\end{array}\right]$,

in which $q$ is the dynamic pressure. The behaviour of both systems is studied by using a parameter which describes the relation between the stiffnesses of the system. Similar to Weisshaar and Ryan (1986) and Weisshaar et al. (1998), the cross-coupling coefficient $\psi$ is defined as:

$\psi=\frac{K_{h \alpha}}{\sqrt{K_{h} K_{\alpha}}}$.

The stiffness matrix has to be positive definite, (van Kan et al. 2005), so the determinant of the stiffness matrix should be larger than zero. Introducing $\psi$ this leads to the following requirement:

$-1<\psi<1$.

The influence of a positive or negative cross-coupling $\Psi$ on the angle-of-attack $\alpha$ versus the dynamic pressure $q$ is depicted in Fig. 3. It can be seen that at increasing dynamic pressure, the angle-of-attack will either increase or decrease for positive or negative bending-torsion coupling, respectively. In the present work, the main interest lies

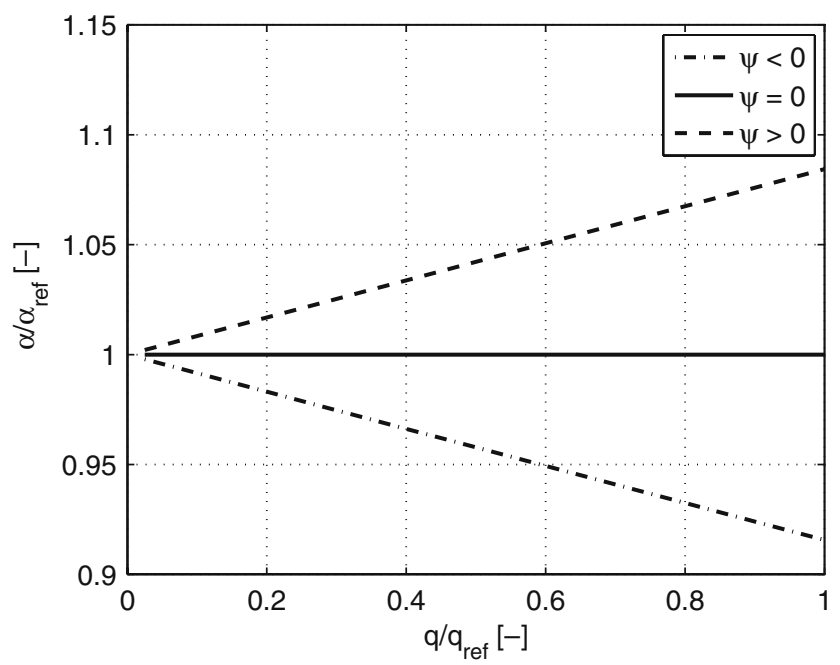

Fig. 3 Influence of cross-coupling $\psi$ on angle-of-attack $\alpha$ at decreasing the angle-of-attack at higher dynamic pressure in order to reduce the induced drag. To facilitate such behaviour, a negative coupling between bending and torsion should be applied.

\section{Three dimensional model}

The two-dimensional analysis has shown that the target behaviour can be achieved when using a negative bendingtorsion coupling $\psi$. According to Katz (1989) low aspect ratio wings have to be designed using a three-dimensional model. Thus a 3D aeroelastic analysis model is developed to investigate the possible drag reduction achievable on an F1 rear wing. This section defines the wing geometry and the corresponding structural and aerodynamic models used in the analysis.

\subsection{Model geometry}

According to the FIA Season 2008 regulations (FIA 2007), the rear wing can consist of two elements, a front and a rear element, as depicted in Fig. 4. By using a multi-element airfoil, the angle-of-attack of the rearward element can be increased, thereby increasing the overall downforce created by the assembly (Katz 1989). In the present study, the rear element is not considered to simplify the model. This simplification is reasonable for an initial proof of concept. The dimensions of the front rear wing element are found in Table 1 (FIA 2007). The wing is assumed to be attached to the car via central pillars, as shown in Fig. 4. This arrangement maximises the flexibility of the wing, making aeroelastic tailoring more effective.

To perform the 3D analysis, a wing cross-section should be known. In F1 racing, an airfoil is chosen for its property of being a high lift/low drag airfoil (Liebeck 1978; Katz 1989). Exact data about present F1 airfoils is hard to find, however, some information is known about airfoils used in the past. One series of airfoils which were often used in F1, and nowadays still are used for airfoil optimisation, are the Benzing airfoils (Benzing 1992), designed by the Italian engineer Enrico Benzing. The chosen airfoil from the

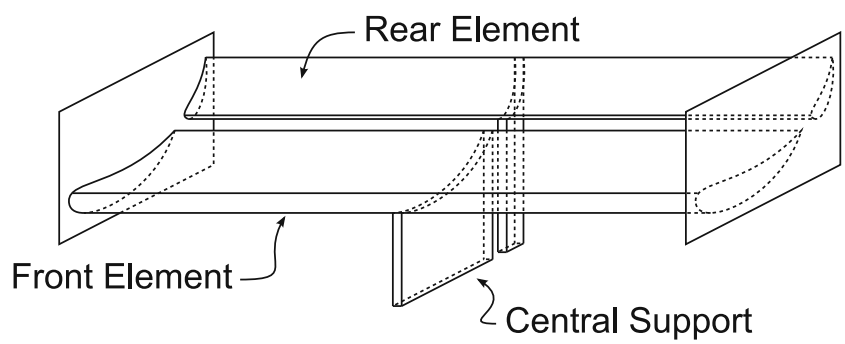

Fig. 4 Support method of the rear wing 
Table 1 Dimensions of F1 rear wing

\begin{tabular}{lll}
\hline Name & Value & Dimension \\
\hline $\mathrm{b}$ & 1 & $\mathrm{~m}$ \\
$\mathrm{c}$ & 0.2 & $\mathrm{~m}$ \\
Taper ratio & 1 & - \\
$R$ & 5 & - \\
\hline
\end{tabular}

Benzing family is known as BE153-105. ${ }^{2}$ A cross section of the BE153-105 airfoil is found in Fig. 5.

\subsection{Structural model}

The structural model used in the 3D analysis consists of several components, each of which are depicted in the schematic overview of the wing in Fig. 6. The inner voids of the wing are filled with a structural foam which is not depicted in the schematic overview. This foam is represented in the discrete model using a solid mesh as explained later in this section. The normal directions of the upper and lower skin are defined such that a CAS lay-up is created when the upper and lower skin are modelled using the same material properties.

All components are given constant material properties, except for the upper and lower skin of the torsion box, which are described using lamination parameters. Symmetric laminates are used and the skin is assumed to be thin enough to be modelled as a membrane. The in-plane stiffness matrix $\mathbf{A}$ is expressed in terms of the lamination parameters:

$\mathbf{A}=h\left(\Gamma_{0}+V_{1} \Gamma_{1}+V_{2} \Gamma_{2}+V_{3} \Gamma_{3}+V_{4} \Gamma_{4}\right)$,

with the stiffness invariants $\Gamma$, defined in terms of the laminate invariants (Gürdal et al. 1999; Diaconu et al. 2002). The four in-plane lamination parameters are defined as:

$$
\left(\begin{array}{l}
V_{1} \\
V_{2} \\
V_{3} \\
V_{4}
\end{array}\right)=\int_{-\frac{1}{2}}^{\frac{1}{2}}\left(\begin{array}{c}
\cos 2 \theta(\bar{z}) \\
\sin 2 \theta(\bar{z}) \\
\cos 4 \theta(\bar{z}) \\
\sin 4 \theta(\bar{z})
\end{array}\right) \mathrm{d} \bar{z}
$$

with $\bar{z}=z / h$ the normalised coordinate in thickness direction, $h$ the total thickness of the laminate, and $\theta(\bar{z})$ the fibre angle at $\bar{z}$.

The wing is modelled in Nastran (MacNeal 1972). Using appropriate symmetry conditions, only half of the actual wing has to be modelled, as shown in Fig. 7. The mesh uses

\footnotetext{
${ }^{2}$ The first 2 digits refer to the maximum relative thickness of the airfoil, the third digit refers to the position of this maximum thickness. The fourth and fifth digit denote the maximum chamber, while the last digit refers to the position of this maximum chamber along the airfoil.
}

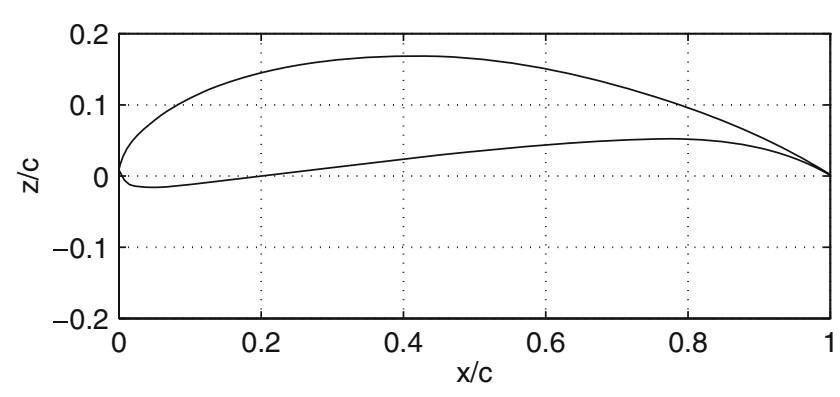

Fig. 5 Cross-section of the BE153-105 airfoil

both rectangular (CQUAD4) and triangular (CTRIA3) elements for meshing the skin, spars, and endplate. The inner solid mesh consists of both five sided (CPENTA) and six sided (CHEXA) solid elements.

\subsection{Aerodynamic model}

An aerodynamic model is necessary to perform Computational Fluid Dynamics (CFD) calculations. The pressure forces are calculated by VSAERO (Maskew 1982), a commercially available panel code capable of calculating the non-linear characteristics of arbitrary objects. The use of a panel code for aerodynamic modelling has two limitations. First, viscous effects can only be taken into account using a post-processing step within VSAERO. It was found that the effect of viscosity is not significant, and results with viscosity are not presented in this paper. Second, an initial wake position has to be provided to VSAERO to be used in its iterative wake relaxation routine.

A representation of the entire aerodynamic model including the wake can be found in Fig. 8. A structured mesh is applied to the wing skin and the trailing edge of the endplate. The remaining part of the endplate is meshed with an unstructured mesh. The input for VSAERO is created using a Matlab routine, which uses the Matlab program DistMesh by Persson and Strang (2004) to create the unstructured mesh.

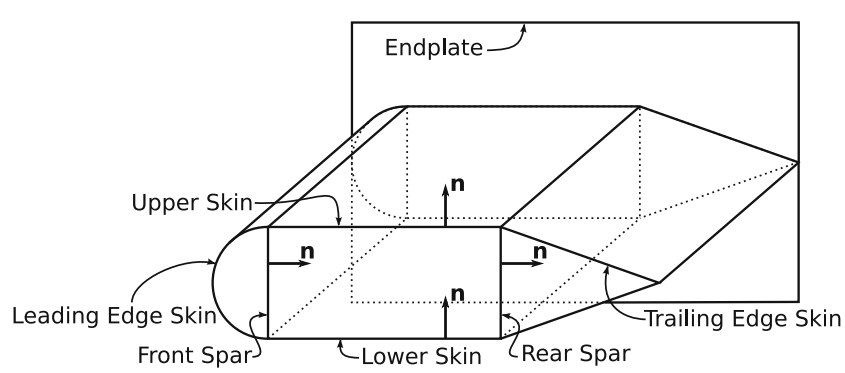

Fig. 6 Schematic overview of wing components 


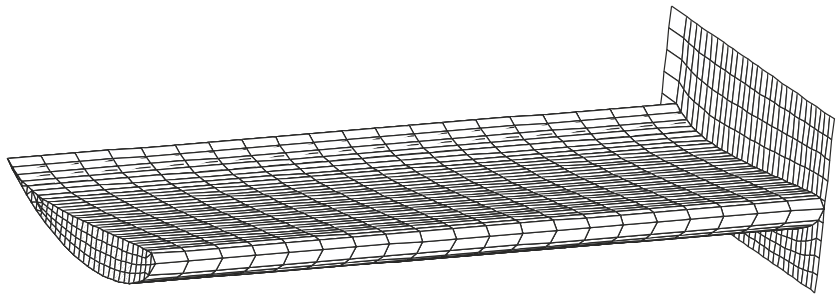

Fig. 7 Structural model of F1 wing with BE153-105 airfoil

\section{Wing simulation and optimisation}

For the purpose of analysing and optimising the 3D wing model, a multi-disciplinary routine is created in ModelCenter, coupling the commercial FE code Nastran to the CFD panel code VSAERO trough a fluid-structure interaction controlled by Matlab routines. The first section discusses the aeroelastic analysis, while the second section highlights the applied fluid-structure coupling. In the third section the optimisation formulation is presented, and the last section explains the implementation in ModelCenter.

\subsection{Aeroelastic analysis}

This work makes use of two commercial solvers, thus a partitioned method, as used by Farhat and Lesionne (1998) is applied to create the aeroelastic analysis. In a partitioned method, the coupling between fluid and structure can be done either by advancing both meshes parallel or serial. The present work uses the serial advancing of both meshes, also referred to as the loosely coupled procedure.

The most basic and common way to introduce this loose coupling is by using the Conventional Serial Staggered (CSS) procedure (Farhat and Lesionne 1998; Beckert 2000). The procedure for this scheme (see Fig. 9) starts by calculating the aerodynamic forces on the undeformed structure (step 1). Next, these aerodynamic forces are transformed to the structural mesh (step 2) to be able to calculate the deflection of the structure (step 3). This deflection is used to update the aerodynamic mesh (step 4) such that the entire procedure can restart on the deformed mesh.

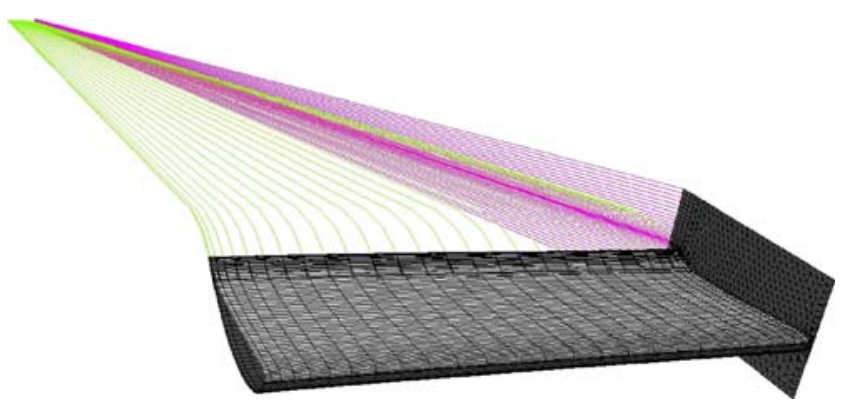

Fig. 8 Aerodynamic model of F1 wing with BE153-105 airfoil
Fluid

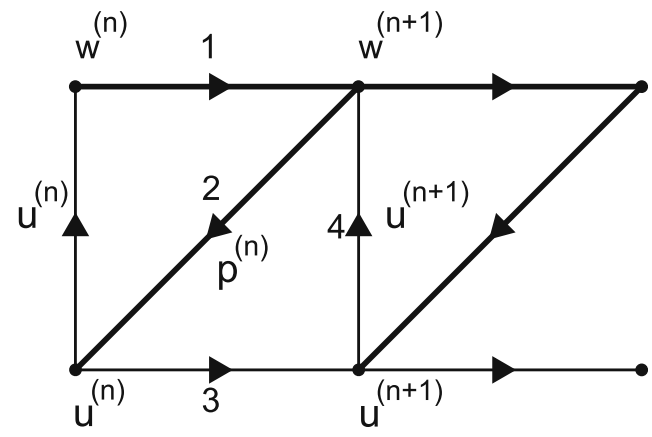

Fig. 9 The Conventional Serial Staggered procedure

\subsection{Fluid-structure coupling}

The structural and aerodynamic model have different meshes. A coupling procedure should be provided to enable the interpolation of forces and displacements between both models. The coupling method used in the present work is based on the principle of equivalence of work. The virtual work done by the aerodynamic loads acting on the aerodynamic model should be equal to the virtual work done by the equivalent aerodynamic loads on the structural model, thereby assuring conservation of energy. According to Beckert (2000) and Beckert and Wendland (2001), a coupling matrix $\mathbf{H}$ can be defined such that:

$\mathbf{u}_{f}=\mathbf{H} \cdot \mathbf{u}_{s}$,

$\mathbf{f}_{s}=\mathbf{H}^{T} \cdot \mathbf{f}_{f}$,

with $\mathbf{u}_{s}$ and $\mathbf{u}_{f}$ the displacement of the structure and fluid boundary, respectively, and $\mathbf{f}_{s}$ and $\mathbf{f}_{f}$ the aerodynamic forces on the structural and aerodynamic model, respectively.

The coupling matrix $\mathbf{H}$ can be constructed using a radial basis function. According to Rendall and Allen (2007), a decaying radial basis function should be chosen. These functions put more emphasis on the local influence, leading to a more physically meaningful force transfer. Moreover, to reduce the computational effort, a compactly supported radial basis function should be applied. The radial basis function used in this work satisfies both requirements, and is known as Wendland's $C^{2}$-function (Wendland 1995; de Boer et al. 2007).

\subsection{Optimisation formulation}

An optimisation is performed to be able to create a rear wing which passively reduces its induced drag at higher velocity. However, the wing should still be able to provide the required downforce to be able to pass a low speed turn. The idea is that the initial fixed angle-of-attack of the wing is chosen such that the wing is able to provide the required 
downforce in low speed turns. By using a bending-torsion coupling, at a higher velocity, the wing should reduce its angle-of-attack as much as possible, thereby reducing the induced drag at this higher velocity.

Thus, the objective for the optimisation is to minimise the induced drag from the wing, calculated by VSAERO, by using the lamination parameters $V_{1}, V_{2}, V_{3}$ and $V_{4}$ as design variables. Since the lamination parameters are defined in terms of trigonometric functions in (14), they are bounded by constraints (Fukunaga and Sekine 1992; Miki and Sugiyama 1993). In order to find a physically meaningful combination of in-plane lamination parameters, the following requirements (Setoodeh et al. 2005) are used as constraints:

$$
\begin{aligned}
& 2 V_{1}^{2}\left(1-V_{3}\right)+2 V_{2}^{2}\left(1+V_{3}\right)+V_{3}^{2}+V_{4}^{2}-4 V_{1} V_{2} V_{4} \leq 1, \\
& V_{1}^{2}+V_{2}^{2} \leq 1 \\
& -1 \leq V_{i} \leq 1, \text { for } i=1,2,3,4
\end{aligned}
$$

\subsection{Implementation in ModelCenter}

ModelCenter (Phoenix Integration 2003) is a process integration environment enabling design and control using various programs from within one visual environment. The flow diagram of the aeroelastic routine using the CSS procedure is depicted in Fig. 10. The initial CFD and Finite Element Model (FEM) meshes are created first. Using these

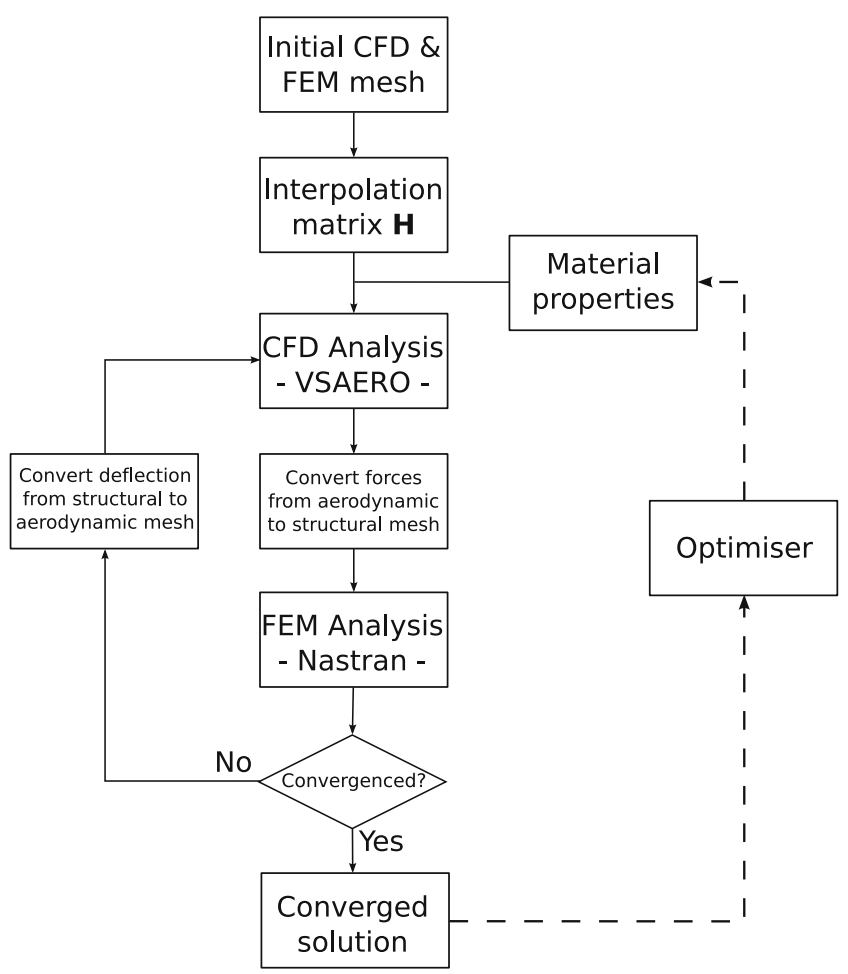

Fig. 10 Flow diagram of 3D aeroelastic routine meshes, the interpolation matrix $\mathbf{H}$, which is independent of any mesh deformation, can be calculated. The material properties are defined by the optimiser. These steps are completed before entering the iterative process, which consists of the CSS procedure, coupling Nastran to VSAERO. A displacement convergence check is performed to decide on the convergence of the fluid-structure iteration.

The optimisation is performed using Design Explorer (Cramer and Gablonsky 2004), a surrogate-model-based optimiser available in ModelCenter. Since no gradient information is required by the optimiser, it is highly suitable for usage with commercial codes such as Nastran and VSAERO.

The Design Explorer will start by creating initial surrogate models based on an initial set of design variables. Using a sequential approximate optimisation method, these surrogate models are used to perform several local optimisations from different starting points. The local optimisation points and some additional points are then evaluated using the actual model. This procedure is denoted as the "Search Step" within Design Explorer. After such a search step, either the information from the actual model evaluations is used to update the surrogate models, or a local exploration around a base point is performed with the actual model ("Poll Step").

\section{Results}

In this section, the output of the optimisation is presented. The first section discusses the optimisation results. The second section considers the results of a Multi-BodySimulation.

\subsection{Optimisation results}

The optimisation is performed for three cases, where the upper and lower skin have a thickness of either 2, 3 or 4 layers. A single layer carbon fibre ply has a thickness of $0.075 \mathrm{~mm}$. During the optimisation, the wing is simulated at an angle-of-attack of 10 degrees, and at a velocity of $92 \mathrm{~m} / \mathrm{s}$.

For the 2 layer model optimisation, a total of 113 function evaluations are performed, where each function evaluation needed typically 3 fluid-structure iterations to converge. However, 42 of the function evaluations have a set of

Table 2 Optimal lamination parameters for 2/3/4 layer models

\begin{tabular}{lllll}
\hline $\mathrm{N}^{\circ}$ layers & $\mathrm{V} 1[-]$ & $\mathrm{V} 2[-]$ & $\mathrm{V} 3[-]$ & $\mathrm{V} 4[-]$ \\
\hline 2 & 0.40625 & -0.61328 & 0.15625 & -0.76563 \\
3 & -0.23437 & -0.56250 & 0.27734 & -0.03516 \\
4 & -0.13282 & -0.18359 & 0.35938 & -0.80078 \\
\hline
\end{tabular}




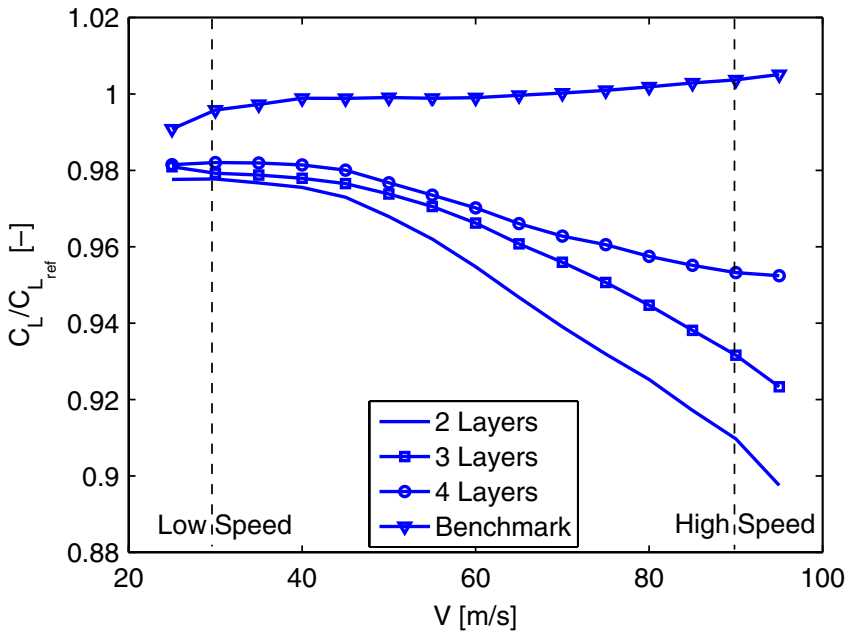

(a)

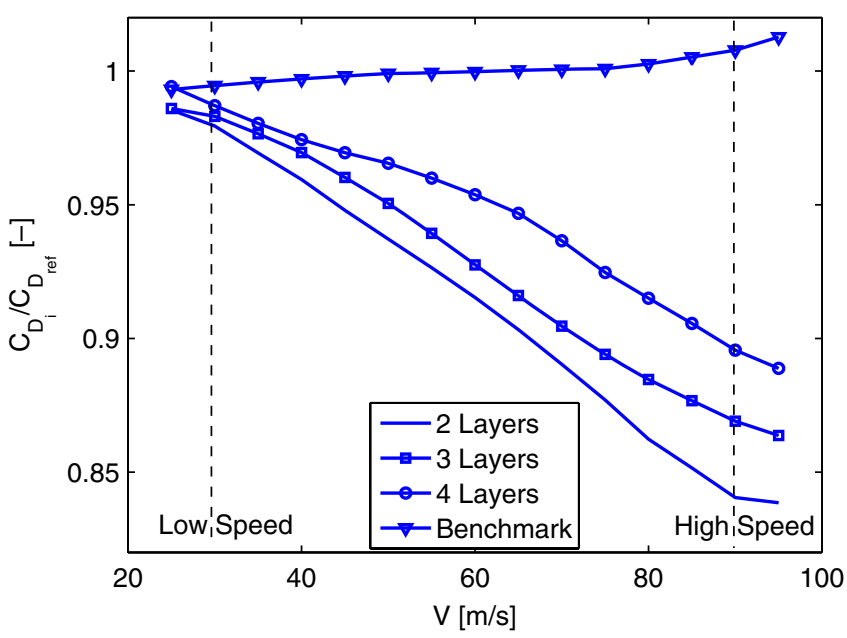

(b)

Fig. 11 Results 2/3/4-layer optimised wing $[\alpha=0.1745$ rad]. a Non-dimensional lift coefficient versus velocity. b Non-dimensional drag coefficient versus velocity

design variables which violate the constraints as defined by (17)-(19). Therefore no actual function evaluation at these design points is performed. Thus 71 runs are performed inside the design space, consisting of 14 search steps and 3 poll steps.

The optimal in-plane lamination parameters for the models having either 2, 3, or 4 layers are given in Table 2. For each of the optimised models, the lift coefficient $C_{L}$ and induced drag coefficient $C_{D_{i}}$ can be plotted against velocity. Comparison of the lift and induced drag coefficient, respectively, against the results for a benchmark wing without bending-torsion coupling are shown in Fig. 11a and b. It can be observed that both the lift and induced drag decrease at higher velocity. This is a direct effect of the wing torsion, which is larger at higher velocity. The torsion induces

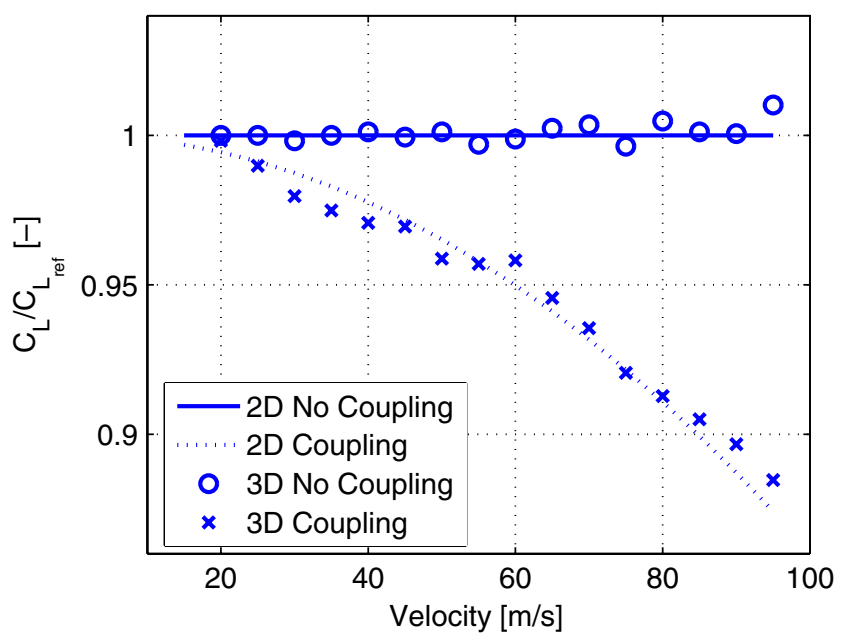

Fig. $123 \mathrm{D}$ versus $2 \mathrm{D}$ comparison of non-dimensionalised lift coefficient a reduction in angle-of-attack, thereby reducing the lift and associated induced drag. By increasing the number of layers, the drag reduction at $90 \mathrm{~m} / \mathrm{s}$ reduces from about $16 \%$ for the 2 layer model to $10 \%$ for the 4 layer model. This is due to the increase of the bending stiffness of the wing by adding layers, thereby decreasing the amount of torsion through the bending-torsion coupling. A lower reduction in angle-of-attack leads to a smaller reduction of the induced drag.

It is worthwhile to investigate whether the relation found by the $2 \mathrm{D}$ analysis is continued by the results from the $3 \mathrm{D}$ analysis. Therefore, the non-dimensional lift coefficients for both 3D optimised and benchmark wing (2 layers) are compared in Fig. 12 with the results obtained from a typical section analysis. From the figure it can be observed that the $3 \mathrm{D}$ result shows good correspondence with the $2 \mathrm{D}$ analysis.

\subsection{Multi-body-simulation}

The influence of the flexible optimised wing on the driving characteristics of a F1 car is investigated in a Multi-Body Simulation (MBS) using the software Simpack (INTEC GmbH 2004). A model having a benchmark wing and a model equipped with the optimised wing are used for two test cases. Both a 10 second acceleration along a straight

Table 3 MBS: acceleration performance

\begin{tabular}{lccl}
\hline Variable & $\begin{array}{l}\text { Benchmark } \\
\text { model }\end{array}$ & $\begin{array}{l}\text { Optimised } \\
\text { model }\end{array}$ & $\begin{array}{l}\text { Absolute } \\
\text { difference }\end{array}$ \\
\hline Distance $[\mathrm{m}]$ & 730.08 & 730.48 & 0.40 \\
Velocity $[\mathrm{m} / \mathrm{s}]$ & 99.65 & 99.79 & 0.14 \\
\hline
\end{tabular}


Table 4 MBS: turn performance

\begin{tabular}{llll}
\hline Variable & $\begin{array}{l}\text { Benchmark } \\
\text { model }\end{array}$ & $\begin{array}{l}\text { Optimised } \\
\text { model }\end{array}$ & $\begin{array}{l}\text { Absolute } \\
\text { difference }\end{array}$ \\
\hline Distance $[\mathrm{m}]$ & 156.45 & 156.48 & 0.03 \\
Velocity $[\mathrm{m} / \mathrm{s}]$ & 29.95 & 29.94 & .01 \\
\hline
\end{tabular}

line, and a $1.8 \mathrm{~g}$ turn at a constant low velocity of $30 \mathrm{~m} / \mathrm{s}$ are investigated. The results for the straight line acceleration are given in Table 3, while in Table 4 the turn performance is presented.

From both tables is can be seen that the optimised model has an advantage during a straight line acceleration, without reducing the turn performance at lower velocity.

\section{Conclusions \& recommendations}

The goal of this work is to find a rear wing for an F1 car capable of passively reducing its angle-of-attack to create a drag reduction at higher velocities. This goal is achieved by using an optimised circumferentially asymmetric stiffness (CAS) lay-up for the torsion box of the rear wing main element. Lamination parameters are applied to describe the variable material properties of the upper and lower skin of this torsion box. These lamination parameters are used as design variables in an aeroelastic optimisation framework, having a minimisation of induced drag as design objective. Based on the results of this optimisation, the following conclusions can be drawn:

- Application of bending-torsion coupling through the use of a CAS lay-up can lead to a substantial drag reduction of an F1 car's rear wing.

- A Multi-Body Simulation (MBS) indicates that the use of the optimised rear wing on an F1 car has an advantage compared to a benchmark car when acceleration in a straight line is considered. The simulation also indicates that the low speed turning performance of the car is maintained.

- The 2D predictions using a typical aeroelastic section show good agreement with the results from the 3D aeroelastic optimisation framework.

- The use of lamination parameters is valuable, since the continuous behaviour of these parameters is useful during optimisation. Once an optimal set of lamination parameters is available, the corresponding lay-up can be determined.

The results show the capability to reduce the induced drag of an F1 car by tailoring the rear wing. When actual application of this concept is considered, current framework can be used, however some updates need to be made to the models:

- The full rear wing assembly needs to be taken into account. This can be done in two ways. The rear element can be considered to be rigid, thus only the aerodynamic influence of it is taken into account while optimising the front element. Another option is to optimise the material properties of both elements simultaneously in order to increase the drag reduction.

- The airfoil type of the rear wing needs to be updated based on more recent data.

- The presence of the car in front of the rear wing needs to be taken into account, as it will have an influence on the airflow acting on the rear wing.

These updates are highly dependent on the actual F1 car and team, since each F1 car has slightly different characteristics with respect to the cars of competing teams. As such, a wing designed for the car of a particular team, might have a different lay-up and performance increase when compared to a wing which is designed for another team's car.

Open Access This article is distributed under the terms of the Creative Commons Attribution Noncommercial License which permits any noncommercial use, distribution, and reproduction in any medium, provided the original author(s) and source are credited.

\section{References}

Abdalla M, De Breuker R, Gürdal Z (2007) Aeroelastic tailoring of variable-stiffness slender wings for minimum compliance. In: IFASD, Stockholm

Abrate S (1994) Optimal design of laminated plates and shells. Compos Struct 29:269-286

Beckert A (2000) Coupling fluid (CFD) and structural (FE) models using finite interpolation elements. Aerosp Sci Technol 4:13-22

Beckert A, Wendland H (2001) Multivariate interpolation for fluidstructure-interaction problems using radial basis functions. Aerosp Sci Technol 5:125-134

Benzing E (1992) Ali/Wings: studio per tecnici e piloti di auto da corsa/study for racing car engineers and drivers. Milano

Bisplinghoff R, Ashley H, Halfman R (1955) Aeroelasticity. AddisonWesley, Reading

de Boer A, van Zuijlen A, Bijl H (2007) Review of coupling methods for non-matching meshes. Comput Methods Appl Mech Eng 196:1515-1525

Bramesfeld G, Ironside D, Schwochow J (2008) Simplified modeling of wing-drag reduction due to structural dynamics and atmospheric gusts. In: 26th AIAA applied aerodynamics conference, Honolulu

Cramer E, Gablonsky J (2004) Effective parallel optimization of complex computer simulations. In: 10th AIAA/ISSMO multidisciplinary analysis and optimization conference, Albany

Diaconu C, Sato M, Sekine H (2002) Feasible region in general design space of lamination parameters for laminated composites. AIAA J 40(3):559-565 
Dowell E (2004) A modern course in aeroelasticity. Kluwer, Dordrecht

Farhat C, Lesionne M (1998) Higher-order staggered and subiteration free algorithms for coupled dynamic aeroelasticity problems. In: 36 th aerospace sciences meeting \& exhibit, Reno

FIA (2007) 2008 Formula 1 technical regulations. Federation Internationale de l'Automobile

Fukunaga H, Sekine H (1992) Stiffness design method of symmetric laminates using lamination parameters. AIAA J 30(11):27912793

Fukunaga H, Sekine H (1994) A laminate design for elastic properties of symmetric laminates with extension-shear of bending-twist coupling. J Compos Mater 28(8):708-731

Fukunaga H, Vanderplaats G (1991) Stiffness optimization of orthotropic laminated composites using lamination parameters. AIAA J 29(4):641-646

Guo S, Cheng W, Cui D (2005) Optimization of composite wing structures for maximum flutter speed. In: 46th AIAA/ASME/ ASCE/AHS/ASC structures, structural dynamics \& materials conference, Austin

Guo S, Cheng W, Cui D (2006) Aeroelastic tailoring of composite wing structures by laminate layup optimization. AIAA J 44(12): 3146-3149

Gürdal Z, Olmedo R (1993) In-plane response of laminates with spatially varying fiber orientations: variable stiffness concept. AIAA J 31(4):751-758

Gürdal Z, Haftka R, Hajela P (1999) Design and optimization of laminated composite materials. Wiley, New York

INTEC GmbH (2004) Simpack user's guide

Kameyama M, Fukunaga H (2007) Optimum design of composite plate wings for aeroelastic characteristics using lamination parameters. Comput Struct 85:213-224

van Kan J, Segal G, Vermolen F (2005) Numerical methods in scientific computing. VSSD

Katz J (1989) Aerodynamics of high-lift, low-aspect-ratio unswept wings. AIAA J 27(8):1123-1124

Katz J (2006) Aerodynamics of race cars. Annu Rev Fluid Mech $38: 27-63$

Liebeck R (1978) Design of subsonic airfoils for high lift. J Aircraft 15(9):547-561

Liu B, Haftka R (2004) Single-level composite wing optimization based on flexural lamination parameters. Struct Multidiscipl Optim 26:111-120

Lynch R, Rogers W (1976) Aeroelastic tailoring of composite materials to improve performance. In: 17th AIAA/ASME/ASCE/ AHS/ASC structures, structural dynamics \& materials conference, King of Prussia, pp 61-68

MacNeal RH (1972) The Nastran theoretical manual. NASA (SP221(01))

Maskew B (1982) VSAERO A computer program for calculating the nonlinear aerodynamic characteristics of arbitrary configurations. NASA (CR-166476)

Massegur D, Quaranta G, Cavagna L (2007) Race cars flex their muscle. ANSYS Advantage 1(1):9-11

Miki M, Sugiyama Y (1993) Optimum design of laminated composite plates using lamination parameters. AIAA J 31(5):921-922
Patil M (1997) Aeroelastic tailoring of composite box beams. In: 35th AIAA aerospace sciences meeting \& exhibit, Reno

Persson P, Strang G (2004) A simple mesh generator in matlab. SIAM Rev 46(2):329-345

Phoenix Integration (2003) Improving the engineering process with software integration

Rehfield L, Cheung R (2003) Some basic strategies for aeroelastic tailoring of wings with bend-twist coupling: part one. In: 44th AIAA/ASME/ASCE/AHS/ASC structures, structural dynamics \& materials conference, Norfolk

Rehfield L, Cheung R (2004) Strategies for aeroelastic tailoring of wings with bend-twist coupling: part two. In: 45th AIAA/ASME/ASCE/AHS/ASC structures, structural dynamics $\&$ materials conference, Palm Springs

Rendall T, Allen C (2007) Unified fluid-structure interpolation and mesh motion using radial basis functions. Int $\mathrm{J}$ Numer Methods Eng

Setoodeh S, Abdalla M, Gürdal Z, Tatting B (2005) Design of variable-stiffness composite laminates for maximum in-plane stiffness using lamination parameters. In: 46th AIAA/ASME/ ASCE/AHS/ASC structures, structural dynamics \& materials conference, Austin

Setoodeh S, Abdalla M, Gürdal Z (2006) Design of variable-stiffness laminates using lamination parameters. Composites: Part B 37:301-309

Shirk M, Hertz T, Weisshaar T (1986) Aeroelastic tailoring-theory, practise, and promise. J Aircraft 23(1):6-18

Stanford B, Ifju P (2008) Aeroelastic tailoring of fixed membrane wings for micro air vehicles. In: 49th AIAA/ASME/ASCE/ AHS/ASC structures, structural dynamics, and materials conference, Schaumberg, IL

Thuwis G, De Breuker R, Abdalla M (2008) Aeroelastic tailoring of a Formula One car rear wing. In: Proceedings of the sixth international conference on engineering computational technology, Athens

Török J (2000) Analytical mechanics. Wiley, New York

Tsai S, Pagano N (1968) Invariant properties of composite materials. In: Composite materials workshop, pp 233-253

Weisshaar T (1981) Aeroelastic tailoring of forward swept composite wings. J Aircraft 18(8):669-676

Weisshaar T, Duke D (2006) Induced drag reduction using aeroelastic tailoring with adaptive control surfaces. J Aircraft 43(1): $157-164$

Weisshaar T, Ryan R (1986) Control of aeroelastic instabilities through stiffness cross-coupling. J Aircraft 23(2):148-155

Weisshaar T, Nam C, Batista-Rodriguez A (1998) Aeroelastic tailoring for improved UAV performance. In: 47th AIAA/ASME/ASCE/ AHS/ASC structures, structural dynamics \& materials conference, Long Beach

Wendland H (1995) Piecewise polynomial, positive definite and compactly supported radial functions of minimal degree. Adv Comput Math 4:389-396

Wright P (1974) Aerodynamics for Formula 1. Aeronaut J 78:226-230

Zhang X, Toet W, Zerihan J (2006) Ground effect aerodynamics of race cars. Appl Mech Rev 59:33-49 